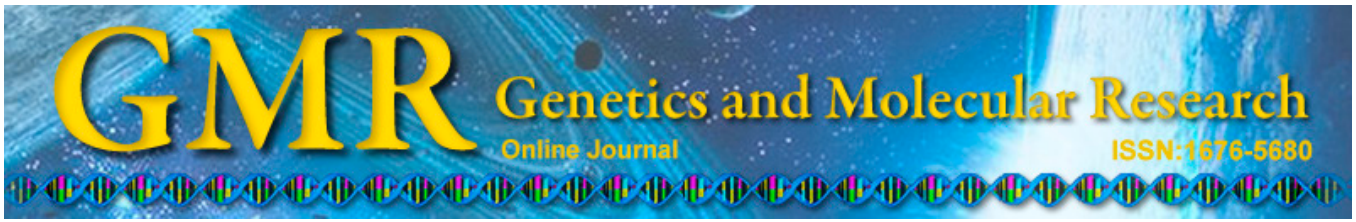

\title{
Effects of hydroxycamptothecin on the expression of matrix metalloproteinase-1 (MMP-1), tissue inhibitor of MMP-1, and type I collagen in rats with pulmonary fibrosis
}

\author{
G.-X. Hu, S.-T. Yao, L.-H. Zeng, Y.-Z. Peng and J. Zheng \\ Peking University Shenzhen Hospital, Shenzhen, China \\ Corresponding author: J. Zheng \\ E-mail: zhengj_zj@163.com
}

Genet. Mol. Res. 14 (2): 4625-4632 (2015)

Received July 21, 2014

Accepted October 28, 2014

Published May 4, 2015

DOI http://dx.doi.org/10.4238/2015.May.4.21

\begin{abstract}
The aim of this study was to investigate the effects of hydroxycamptothecin (HCPT) on the expression of matrix metalloproteinase-1 (MMP-1), tissue inhibitor of MMP-1 (TIMP-1), and type I collagen in the lung tissue of rats with pulmonary fibrosis induced by bleomycin A5. We used hematoxylin eosin staining to observe the degree of pulmonary fibrosis in rats; Masson staining, reverse transcription polymerase chain reaction, and immunohistochemistry were used to observe the expression of collagen, MMP-1, and TIMP1 , and type I collagen. The expression of MMP-1 in the model group decreased significantly, while the expression of TIMP-1 and type I collagen significantly increased. After treatment with HCPT, the degree of pulmonary fibrosis and the expression of TIMP-1 and type I collagen decreased in all treatment groups. However, the expression of MMP-1 increased in a dose-dependent manner. Our results showed that HCPT decreased the pulmonary fibrosis induced by bleomycin A5 in rats,
\end{abstract}


and an increase in MMP-1 expression and decrease in the TIMP-1 and type I collagen expression may be the mechanism that regulates the metabolism of the extracellular matrix.

Key words: Pulmonary fibrosis; Hydroxycamptothecin; Type I collagen; Matrix metalloproteinase-1; Matrix metalloproteinase inhibitors-1

\section{INTRODUCTION}

Pulmonary fibrosis is an alveolar interstitial inflammatory disease caused by many factors. This disease is characterized by proliferation of fibroblasts and aggregation of the extracellular matrix (ECM). The incidence of pulmonary fibrosis has gradually increased in recent years, and the conventional treatment for this disease in Western medicine includes administration of adrenal cortical hormone and immunosuppressive agents. However, this treatment is not very effective and is associated with side effects; the five-year survival rate of patients receiving this treatment is only 50\% (Xiao and Gao, 2007; Hourong, 2010). Hydroxycamptothecin (HCPT) is a camptothecin analogue extracted from the Chinese plant Camptotheca acuminata and has anti-tumor activity. Recent studies show that HCPT inhibits proliferation and induces apoptosis of various cells that cause fibrosis in different tissues and organs (Qian et al., 2006; Liao et al., 2010). However, the effect of HCPT on pulmonary fibrosis has not been clarified to date. In this study, we investigated the effects of HCPT on the expression of matrix metalloproteinase-1 (MMP-1), tissue inhibitor of MMP-1 (TIMP-1) and collagen type I, which may provide insight into the clinical implications of HCPT in the treatment of pulmonary fibrosis.

\section{MATERIAL AND METHODS}

\section{Materials}

\section{Subjects}

We used clean-grade Sprague-Dawley $(\mathrm{SD})$ rats $(\mathrm{N}=50$; male $=25$, female $=25)$ weighing 180-220 g. The rats were purchased from the animal experimental center of Jiangxi University of Traditional Chinese Medicine [production license number: SCXK (Jiangxi) 2005-0001] and were fed and maintained in a clean experimental cage ( $\mathrm{N}=5$ in each cage). The animals were fed $25 \mathrm{~g}$ /day normal diet and given plenty of pure water. The temperature in the animal room was $22-25^{\circ} \mathrm{C}$ and $50 \%$ relative humidity with natural light.

\section{Main reagents}

We used bleomycin hydrochloride A5 (Tianjin Taihe Pharmaceutical Co. Ltd.), HCPT (Huangshi Li Shizhen Pharmaceutical Group Pharmaceutical Co., Ltd., Wuhan Li Shizhen), reverse transcription polymerase chain reaction (RT-PCR) Kit (Promega, USA), MMP-1 and TIMP-1 primers (SANGON Biological Engineering Co., Ltd., Shanghai, China), rabbit antirat collagen type I polyclonal antibody (Santa Cruz Biotechnology, USA). 


\section{Methods}

\section{Grouping}

Fifty rats were randomly divided into five groups (control group, model group, lowdose group, middle-dose group, and high-dose group) with 10 rats in each group.

\section{Operation}

A rat model of pulmonary fibrosis was developed by intratracheal injection of bleomycin A5. The rats were anesthetized, the trachea was exposed, and an intratracheal injection of $5 \mathrm{mg} / \mathrm{mL}$ bleomycin A5 solution at $0.1 \mathrm{~mL} / 100 \mathrm{~g}$ (weight) was administered into a $1-\mathrm{cm}$ thick section of the neck by transverse incision; the animals were rotated rapidly after injection.

\section{Administration}

HCPT was diluted to a $0.1 \mathrm{mg} / \mathrm{mL}$ solution, and an intraperitoneal injection was administered according to the low $(0.5 \mathrm{mg} / \mathrm{kg})$, middle $(1.0 \mathrm{mg} / \mathrm{kg})$, and high $(1.5 \mathrm{mg} / \mathrm{kg})$ doses, while the control group and model group were administered the same volumes of normal saline $(2 \mathrm{~mL} /) 3$ times a week for 4 weeks.

\section{Preparation of lung tissue}

Twenty-eight days after preparation of the rat model, the rats were killed by exsanguination from the abdominal aorta. The right upper section of the lung was soaked in $10 \%$ neutral formalin solution, and the remaining tissue was stored in liquid nitrogen.

\section{Detection and examination of the lung tissue}

The right upper lung tissue was fixed in $10 \%$ neutral formaldehyde solution for $24 \mathrm{~h}$; subsequently, paraffin sections were used to perform hematoxylin and eosin (H\&E) staining, Masson staining, and immunohistochemical analysis.

Alveolitis was evaluated after H\&E staining according to the Szapiel method (Szapiel et al., 1979). The specific grading standards were as follows: 1) no fibrosis, 0 points; 2) range of lesion $<20 \%, 1$ point; 3) range of lesion from 20 to 50\%, 2 points; and 4) range of lesion $>50 \%, 3$ points.

After Masson staining, the images were analyzed using the Image-pro Plus professional image analysis software system, and the degree of pulmonary fibrosis was represented as collagen area/lung field (\%) in each section, which was calculated separately from five fields of the periphery and the center.

We randomly selected five fields from the periphery and center of each section; the absorbance value of particles that expressed type I collagen were determined using the SABC immunohistochemical technique and the German VIDAS image analysis system; the average values were used to determine the expression of lung collagen I in rats. 


\section{RT-PCR detection}

Total RNA was extracted from the lung tissue according to the Trizol Kit method. The first strands of cDNA were reversed-transcribed in accordance with the method in the reverse transcription kit, and then, the cDNA was used as the template for the PCR amplification reaction. $\beta$-actin was used as the internal reference. The primer sequences were as follows: $M M P$ 1 sense strand 5'-TTGTTGCTGCCCATGAGCTT-3', antisense 5'-ACTTTGTCGCCAATTC CAGG-3'; TIMP-1 sense strand 5'-TTTGCATCTCTGGCCTCTG-3', antisense 5'-AATGACT GTCACTCTCCAG-3'; and $\beta$-actin justice chain 5'-TCAGGTCATCACTACTGGCAAT-3' antisense 5'-AAAGAAAGGGTGTAAAACGCA-3'. The reaction conditions were as follows: $M M P-1$ : initial denaturation at $94^{\circ} \mathrm{C}$ for $5 \mathrm{~min}$, and denaturation at $95^{\circ} \mathrm{C}$ for $45 \mathrm{~s}$, annealing at $60^{\circ} \mathrm{C}$ for $45 \mathrm{~s}$, extension at $72^{\circ} \mathrm{C}$ for $2 \mathrm{~min}$ for 40 cycles, followed by final extension at $72^{\circ} \mathrm{C}$ for $8 \mathrm{~min}$; the final product was $639 \mathrm{bp}$. TIMP-1: initial denaturation at $94^{\circ} \mathrm{C}$ for $5 \mathrm{~min}$, and denaturation at $94^{\circ} \mathrm{C}$ for $15 \mathrm{~s}$, annealing at $60^{\circ} \mathrm{C}$ for $30 \mathrm{~s}$, extension at $72^{\circ} \mathrm{C}$ for $30 \mathrm{~s}$ for 40 cycles, followed by final extension at $72^{\circ} \mathrm{C}$ for $8 \mathrm{~min}$; the final product was $495 \mathrm{bp}$; $\beta$-actin: denaturation at $94^{\circ} \mathrm{C}$ for $5 \mathrm{~min}$, and denaturation at $94^{\circ} \mathrm{C}$ for $35 \mathrm{~s}$, annealing at $52^{\circ} \mathrm{C}$ for 35 $\mathrm{s}$, extension at $72^{\circ} \mathrm{C}$ for $35 \mathrm{~s}$ for a total of 30 cycles, followed by final extension at $72^{\circ} \mathrm{C}$ for $7 \mathrm{~min}$; the end product was $425 \mathrm{bp}$. The PCR products were analyzed using $1 \%$ agarose gel electrophoresis, and analyzed on a gel imaging system using the absorbance measured relative to the $\beta$-actin product absorbance.

\section{Statistical analysis}

The data were analyzed using the statistical software SPSS15.0. The data are reported as means \pm standard deviation. The Student $t$-test was used to compare two samples and the F test was used to compare the mean values of five groups.

\section{RESULTS}

\section{Analysis of lung tissue morphology}

\section{Gross observation}

Both the lungs of the rats in the control group were pink, had a smooth surface, and were elastic. However, both the lungs of the rats in the model group were pale, had a rough surface, a visible bleeding point, and a large ecchymosis. Compared to the model group, the groups receiving HCPT showed a dose-dependent improvement in the volume, color, elasticity, and qualitative hard and nodular changes in both the lungs.

\section{Analysis of the lung tissue after HE and Masson staining}

The lung tissue of rats in the control group was clear and no collagen fiber distribution was observed under the light microscope. The rats in the model group showed damage to the alveolar structure and many blue green collagen fibers surrounding the lesions. Compared to the model group, the groups receiving HCPT showed a dose-dependent decrease in the damage to the alveolar wall and collagen distribution $(\mathrm{P}<0.05$; Table 1, Figure 1, Figure 2$)$. 
Table 1. Comparison of alveolitis and pulmonary fibrosis score in each group $(\mathrm{N}=10$, means $\pm \mathrm{SD})$.

\begin{tabular}{|c|c|c|}
\hline Group & Alveolitis & Pulmonary fibrosis \\
\hline Control group & $0 \pm 0$ & $0 \pm 0$ \\
\hline Model group & $2.8 \pm 0.42$ & $58.10 \pm 7.46^{\prime}$ \\
\hline Low-dose group & $2.4 \pm 0.70^{=\mathbf{4}}$ & $50.40 \pm 2.17^{-4}$ \\
\hline Middle-dose group & $2.2 \pm 0.63^{\mathbf{A}}$ & $35.30 \pm 2.31^{\mathbf{A 4}}$ \\
\hline High-dose group & $1.5 \pm 0.70^{\boldsymbol{~} \mathbf{\Delta}}$ & $16 .^{20} \pm 2.30^{\mathbf{A}}$ \\
\hline$F$ value & 39.01 & 0.00 \\
\hline$P$ value & 410.3 & 0.00 \\
\hline
\end{tabular}

- Compared to the control group $\mathrm{P}<0.01,{ }^{\wedge}$ Compared to the model group $\mathrm{P}<0.05$.

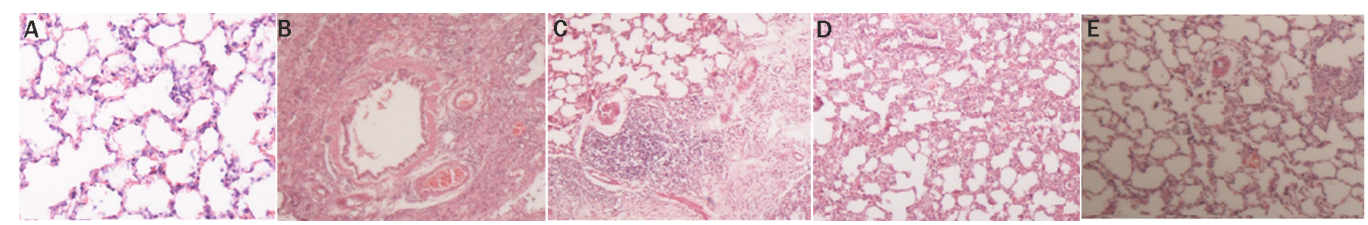

Figure 1. Results of hematoxylin eosin (HE) staining: $(\mathbf{A}=$ Control group; $\mathbf{B}=$ Model group; $\mathbf{C}=$ Low-dose group; $\mathbf{D}=$ Middle-dose group; $\mathbf{E}=$ High-dose group).

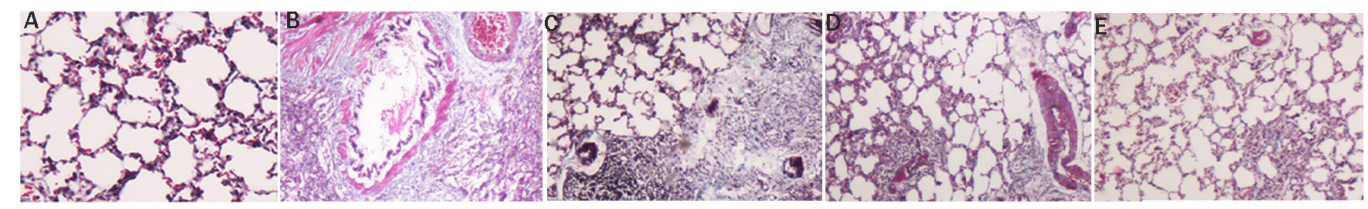

Figure 2. Results of Masson staining: $(\mathbf{A}=$ Control group; $\mathbf{B}=$ Model group; $\mathbf{C}=$ Low-dose group; $\mathbf{D}=$ Middledose group; E High-dose group).

\section{Expression of type I collagen}

Immunohistochemical staining showed weak expression of type I collagen in the organs surrounding the lung tissue in rats in the control group. Rats in the model group showed wide distribution of type I collagen in the bronchial subepithelial tissue and pulmonary interstitial and perivascular spaces. The expression of type I collagen in the HCPT group was lower than that in the model group and showed a dose-dependent decrease (Figure 3, Table 2).

Table 2. Comparison of the absorbance values of collagen type I, matrix metalloproteinase-1 (MMP-1), and tissue inhibitor of MMP-1 (TIMP-1) mRNA ( $\mathrm{N}=10$, means $\pm \mathrm{SD}$ ).

\begin{tabular}{|c|c|c|c|}
\hline Group & Type I collagen & MMP-1 & TIMP-1 \\
\hline Control group & $0.27 \pm 0.004$ & $0.92 \pm 0.04$ & $0.17 \pm 0.004$ \\
\hline Model group & $0.63 \pm 0.02$ & $0.32 \pm 0.008^{*}$ & $2.10 \pm 0.21^{*}$ \\
\hline Low-dose group & $0.62 \pm 0.03=\mathbf{4}$ & $1.27 \pm 0.63^{\mathrm{\Delta} \Delta}$ & $1.89 \pm 0.37^{*}$ \\
\hline Middle-dose group & $0.58 \pm 0.05^{\star \Delta}$ & $1.71 \pm 0.75^{\star \Delta}$ & $1.49 \pm 0.63^{*}$ \\
\hline High-dose group & $0.56 \pm 0.044^{\star \Delta}$ & $2.46 \pm 0.30^{\star \Delta}$ & $1.12 \pm 0.53^{\star \Delta}$ \\
\hline$F$ value & 205.6 & 31.07 & 33.75 \\
\hline $\mathrm{P}$ value & 0.00 & 0.00 & 0.00 \\
\hline
\end{tabular}

-Compared to the control group $\mathrm{P}<0.01,{ }^{\wedge}$ Compared to the model group $\mathrm{P}<0.05,{ }^{\Delta}$ Compared to the model group $\mathrm{P}<0.01$. 


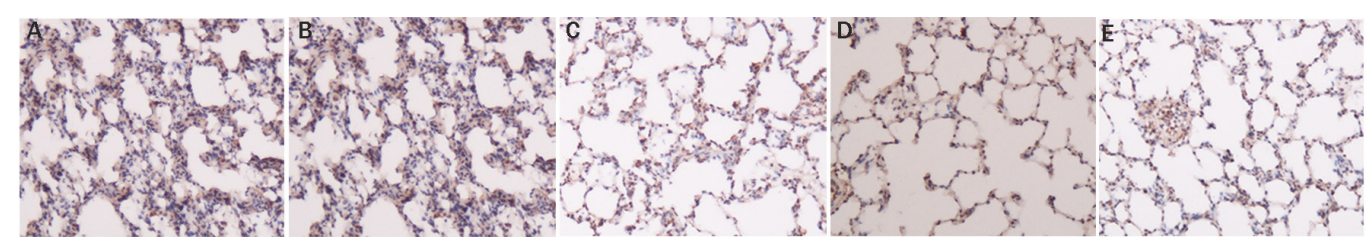

Figure 3. Results of immunohistochemistry ( $\mathbf{A}=$ Control group; $\mathbf{B}=$ Model group; $\mathbf{C}=$ Low-dose group; $\mathbf{D}=$ Middle-dose group; $\mathbf{E}=$ High-dose group).

\section{Expressions of MMP-1 and TIMP-1 mRNA}

Compared to the normal group, the model group showed a decrease in the expression of $M M P-1$ mRNA, while the expression of TIMP-1 mRNA increased. The expression of $M M P-1$ showed a dose-dependent increase, and the expression of TIMP-1 showed a dosedependent decrease after the administration of HCPT $(\mathrm{P}<0.05$; Figure 4 , Table 2$)$.
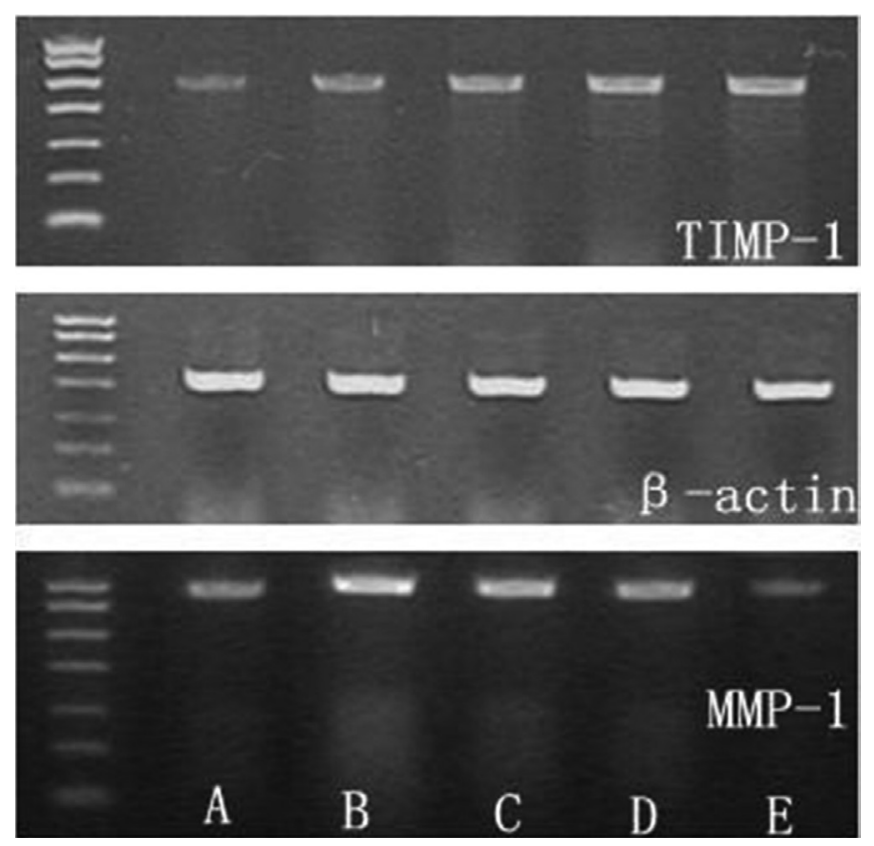

Figure 4. Expression of matrix metalloproteinase-1 (MMP-1) and tissue inhibitor of MMP-1 (TIMP-1) mRNA in each group ( $\mathbf{A}=$ Control group; $\mathbf{B}=$ High-dose group; $\mathbf{C}=$ Middle-dose group; $\mathbf{D}=$ Low-dose group; $\mathbf{E}=$ Model group).

\section{DISCUSSION}

HCPT was extracted from the bark of $C$. acuminata, a plant native to China, which belongs to the Nyssaceae family. HCPT mainly plays a role in the inhibition of cell proliferation, induction of apoptosis, and progression of the cell cycle. A previous in vitro study has shown that HCPT inhibits the proliferation of human embryonic lung fibroblasts and induces apoptosis in a dose- and time-dependent manner (Zheng et al., 2011). In addition, HCPT inhibits the growth of human embryonic lung fibroblasts through inhibition of cell proliferation 
and induction of cell apoptosis in vitro (Jiang et al., 2011). To date, no study has described the in vivo effects of HCPT on pulmonary fibrosis in China.

In this study, we developed a rat model of pulmonary fibrosis by using the internationally recognized method of intratracheal injection of bleomycin A5 to effectively evaluate the efficacy of drugs on pulmonary fibrosis (Li et al., 2009). Our results showed that the lung tissue of rats in the model group appeared pale, showed a decrease in volume, had poor flexibility, qualitative hard surface nodules, and old hemorrhage. HE staining showed damage to the alveolar wall, atrophy, and presence of inflammatory cell infiltrate in the alveolar wall. The fibrous tissue was distributed with patch cords widely. Masson staining showed a large number of blue-green collagen fibers surrounding the bronchi, bronchioles, and small vessels. Collagen hyperplasia and the extent of deposition in the lung interstitium suggested successful induction of pulmonary fibrosis in the rat model. Compared to the model group, the group receiving different dosages of HCPT showed an improvement in the symptoms of paleness, size, degree of elasticity, surface texture, nodular changes, and bleeding in both the lungs. In addition, the alveolitis and fibrosis resolved, and the alveolar wall damage and collagen distribution in each HCPT group decreased, which suggested that HCPT plays a role against alveolitis and pulmonary fibrosis.

The ECM consists of macromolecules, which are secreted by the cell into the ECM. The four main components of the ECM in the lung are collagen, elastin, proteoglycan, and sugar laminin. These ingredients impart a certain degree of hardness and toughness to the tissue, and thus, enable the tissue in its physiological function. Excessive deposition of collagen in the ECM impairs the normal physiological structure and function, and thus, induces pulmonary fibrosis. Collagen is the main component of the ECM; in particular, types I and III collagen were the main interstitial collagen in the lung. In this study, we investigated the effects of HCPT on the expression of type I collagen in rats with pulmonary fibrosis by using an immunohistochemical method. Our results showed that the expression of type I collagen in the bronchial subepithelial tissue and pulmonary interstitial and perivascular spaces increased; however, compared to the model group, the groups receiving HCPT showed a dose-dependent decrease in the expression of type I collagen in those areas. Our results suggested that HCPT can inhibit the secretion of type I collagen in rats with pulmonary fibrosis in a dose-dependent manner. Our results were consistent with those reported by Li et al. (2009).

MMPs/TIMPs are one of the most important groups of enzymes that affect the degradation of the ECM (Chen et al., 2007). The ECM is in a dynamic balance of continuous emergence and degradation under physiological conditions. MMP-1 is a key enzyme that belongs to MMPs, and MMP-1 plays an important role in the degradation of type I collagen, while TIMP-1 is the most active enzyme belonging to the TIMPs, and TIMP-1 plays a role in the inhibition of MMPs, especially MMP-1, and thus, prevents the degradation of the ECM through irreversible binding with the activated MMP-1. A decrease in the ratio of MMP-1/TIMP-1 and degradation of type I collagen results in excess deposition of collagen in the ECM and thus leads to fibrosis (Yuan et al., 2010). In this study, we examined the expression of $M M P-1$ and TIMP-1 mRNA in a rat model of pulmonary interstitial fibrosis by using RT-PCR. Our results showed that a decrease in the expression of $M M P-1$ and an increase in the expression of $T I M P-1$. The expression of $M M P-1$ and TIMP-1 showed a significant dose-dependent increase or decrease independently of each other after treatment with HCPT. Thus, our results showed that HCPT can decrease the synthesis of the ECM collagen by restoring the balance between the MMP/TIMP network and increase the degradation of collagen so as to decrease or inhibit 
pulmonary interstitial fibrosis.

In conclusion, HCPT plays a role in inhibiting pulmonary fibrosis, and HCPT decreased bleomycin A5-induced pulmonary fibrosis in rats. The mechanism underlying this effect may be an increase in MMP-1 expression and a decrease in the TIMP-1 and type I collagen expression that regulate the metabolism of the ECM and decrease or inhibit pulmonary interstitial fibrosis.

\section{ACKNOWLEDGMENT}

Research supported by the National Natural Science Foundation of China (Grant \#30860230); Project supported by the Health and Family Planning Commission of Shenzhen Municipality (Grant \#201401046).

\section{REFERENCES}

Chen P, Wang XJ and Yang F (2007). The roles of matrix metalloproteinases and their inhibitors in the pulmonary fibrosis. Chin. J. Coal Ind. Med. 10:1131-1132.

Hourong C (2010). The confusion and reflection of clinical study of idiopathic pulmonary fibrosis therapy. Chin. J. Tubercul. Respir. Dis. 33: 869-870.

Jiang RJ, Zheng J, Liu LJ, et al. (2011). The effects of HCPT on proliferation and apoptosis of human embryo lung fibroblast. The J. Practical Med. 27: 4364-4366.

Li L, Liao LX, Chen GQ, et al. (2009). The effects of HCPT on secretion of human hypertrophic scar fibroblasts collagen. Acta Acad. Medicinae Jiangxi, 49: 1-3.

Liao LX, Pan D, Chen GQ, et al. (2010). The effects of HCPT on human pathological scar fibroblasts proliferation. Chin. J. Tissue Eng. Res. 14: 6159-6162.

Qian XP, Baorui L, Hu J, Hu WJ, et al. (2006). The study of Shenmai injection combined with hydroxycamptothecin on the inhibition of angiogenesis. China Oncol. 16: 953-957.

Szapiel SV, Elson NA, Fulmer JD, Hunninghake GW, et al. (1979). Bleomycin-induced interstitial pulmonary disease in the nude, athymic mouse. Am. Rev. Respir. Dis. 120: 893-899.

Xiao F and Gao J (2007). Progress in the treatment of idiopathic pulmonary fibrosis. J. Clin. Pulm. Med. 2: 155.

Yuan J, Yang Y, Zhou XY, et al. (2010). Effects of touxietongluo therapy on MMP-1 and TIMP-1 in hepatic tissue of hepatic fibrosis rats. J. Jiangxi Univ. Tradit. Chin. Med. 22: 76-79.

Zheng J, Jiang RJ, Li L, et al. (2011). The effects of HCPT on the growth of human embryo lung fibroblasts HFL1. Shanghai J. Tradit. Chin. Med. 45: 80-83. 